\title{
Dégradation des pesticides organochlorés par les traitements technologiques
}

\author{
par \\ Marie-France GUINGAMP* et Charles ALAIS**
}

\section{INTRODUCTION}

Les vaches métabolisent et rendent inactifs un grand nombre de pesticides, mais laissent presque intacts les composés organcchlorés dont une partie est excrétée dans le lait. Malgré les mesures réglementaires prises dans de nombreux pays, le lait et les produits laitiers contiennent encore ces substances.

D'assez nombreux travaux ont été faits afin de trouver une possibilité de réduire les taux de pesticides organochlorés présents dans le lait ou les produits laitiers. Par exemple, Langlois et al. $[1,2]$ et Li et al. [3] constatent que la transformation du lait en produits laitiers (crème, beurre et fromage) et le stockage de ces produits laitiers, n'ont que peu ou pas d'influence sur les pesticides organochlorés. Seule, la fabrication du lait en poudre (procédé Spray) agit sur ces derniers de façon sensible. Ledford et al. [4] font subir à du lait contaminé un traitement dans un système industriel ( Steam desodorization »), destiné à éliminer certaines flaveurs désagréables ; cette expérience permet de détruire 24 p. 100 de lindane, 8 p. 100 de D.D.T., 3 p. 100 de dieldrine ; l'heptachlore n'est pas touché. Kroger [5] rapporte que 95 p. 100 d'heptachlore époxyde et de dieldrine sont éliminés de la graisse de lait anhydre par un traitement identique mais plus poussé $\left(180-195^{\circ} \mathrm{C} ; 0,5 \mathrm{~mm} \mathrm{Hg} ; 5 \mathrm{~h}\right)$.

Li et Bradley [6] ont étudié la dégradation de plusieurs organochlorés : heptachlore époxyde, T.D.E., perthane, kelthane, D.D.T. et méthoxychlore, par des rayonnements ultra-violets $(220-330 \mathrm{~nm})$. Keith et Alford [7] ont mis en évidence, à l'aide de la R.M.N., l'apparition de photoisomères toxiques, lors de l'irradiation de l'aldrine, de la dieldrine, de l'heptachlore et de l'heptachlore époxyde.

\footnotetext{
* Département de Biologie appliquée, I.U.T. de Nancy.

** Service de Biochimie appliquée, Université de Nancy I.
} 
Hekmati et Bradley [8] ont montré que l'eau oxygénée à une concentration de $0,03 \mathrm{p} .100$ en poids, agissant à $4^{\circ} \mathrm{C}$ pendant $15 \mathrm{mn}$, sur du lait contaminé, permet d'éliminer 38,3 p. 100 de dieldrine, 31 p. 100 de chlordane, 18 p. 100 de lindane, 17 p. 100 d'aldrine, 11 p. 100 d'heptachlore époxyde. Les dégradations seraient dues à une déchlorhydratation.

Dans le travail qui fait l'objet de cette communication, et qui a été commencé en 1970, nous avons étudié la dégradation des principaux pesticides organochlorés soumis à différents traitements du type de ceux que l'on rencontre dans la pratique : traitement thermique, mais plus sévère que celui de la stérilisation ; rayonnements ultra-violets ; traitement à l'eau oxygénée (utilisée dans certains pays et notamment en Amérique pour le lait de fromagerie) et stockage de longue durée.

\section{MATERIELS ET METHODES}

\section{1) Préparation des échantillons}

Dans la plupart des essais, on a utilisé de la graisse de beurre contaminée, à raison de $1 \mathrm{ppm}$, par divers pesticides. Les mélanges sont préparés de manière à éviter les interférences dues à la transformation éventuelle d'un composé en son époxyde.

- Solution $\mathrm{n}^{\circ}$ 1: H.C.H. $\alpha$ + heptachlore + dieldrine. époxyde.

- Solution $n^{\circ} 2$ : H.C.H. $\gamma$ (lindane) + aldrine + heptachlore

A $1 \mathrm{~g}$ de graisse de beurre, on ajoute $1 \mathrm{ml}$ d'une solution (1 ou 2) de pesticides dans l'hexane. Dans le cas du traitement thermique, après évaporation du solvant, le tube est scellé à l'air ambiant.

La dégradation par les rayonnements U.V. se fait dans des coupelles en porcelaine. Pour l'étude de l'effet de l'eau oxygénée, on a utilisé $10 \mathrm{ml}$ de lait pasteurisé contaminé par $1 \mathrm{ml}$ de solution

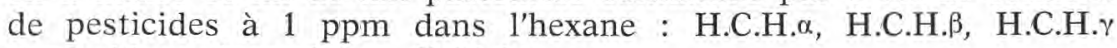
heptachlore époxyde et dieldrine.

\section{2) Traitements subis par les échantillons}

Pour le traitement thermique, les tubes scellés ont été placés dans un four à mouffle à différentes températures : $150^{\circ} \mathrm{C}, 200^{\circ} \mathrm{C}$, $250^{\circ} \mathrm{C}, 300^{\circ} \mathrm{C}$ et $350^{\circ} \mathrm{C}$, pendant des temps variables : $1 \mathrm{~h}, 2 \mathrm{~h}, 3 \mathrm{~h}$, $4 \mathrm{~h}$ et $5 \mathrm{~h}$.

Le rayonnement ultra-violet a été dirigé sur les coupelles contenant la graisse contaminée (rayonnement d'une lampe émettant à $254 \mathrm{~nm}$ ) pendant des durées variant de $1 \mathrm{~h}$ à $5 \mathrm{~h}$. 
L'eau oxygénée a été ajoutée au lait contaminé de façon à avoir une solution à $0,03 \mathrm{p} .100$ en poids. Après $15 \mathrm{mn}$, on a ajouté de la catalase pour détruire l'eau oxygénée.

Pour le stockage, les tubes en verre pyrex, bouchés, ont été abandonnés pendant $12,20,28$ mois à $6^{\circ} \mathrm{C}$ ou à $22^{\circ} \mathrm{C}$, à la lumière ou à l'obscurité.

\section{3) Analyse des échantillons}

Le contenu des tubes ou des coupelles est traité suivant la méthode de Langlois et al. [9]. La détection des résidus des pesticides se fait par chromatographie en phase gazeuse, sur deux appareils Varian Aerograph série 1400, équipés de détecteurs à capture d'électrons (source au tritium) et de colonnes dont les phases stationnaires ont des polarités différentes : $\mathrm{QF}_{1}$ et $\mathrm{DOW}_{11}$.

\section{RESULTATS}

\section{1) Traitement thermique}

Après les traitements à $150^{\circ} \mathrm{C}$ et à $200^{\circ} \mathrm{C}$, les pesticides organochlorés sont peu ou pas dégradés. La figure 1 montre les pourcentages résiduels à $200^{\circ}$ C. Seuls les H.C.H. ont subi une réduction notable; il en reste 75 p. 100 après 5 h de chauffage.

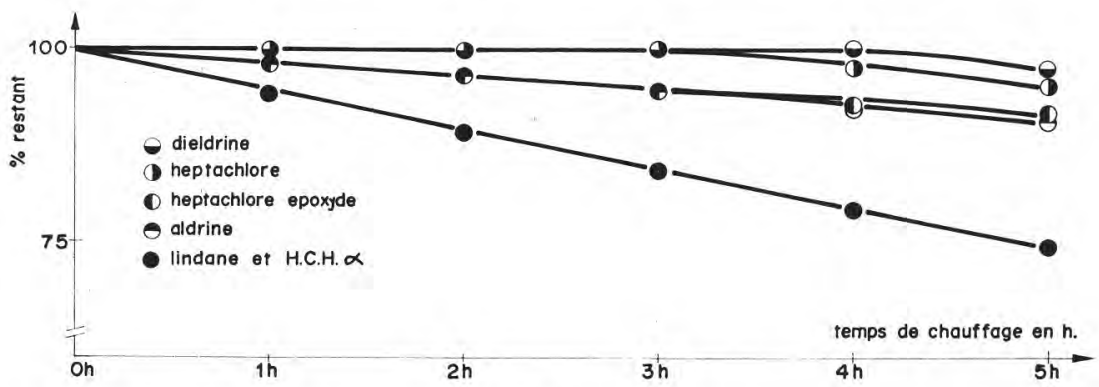

fig. 1

Effets du chauffage $200^{\circ} \mathrm{C}$

La figure 2 présente les résultats obtenus à $300^{\circ} \mathrm{C}$. On voit que les H.C.H. sont réduits à 25 p. 100 en $1 \mathrm{~h}$ et détruits totalement en $3 \mathrm{~h}$. La destruction des autres pesticides organochlorés est moins rapide, la dieldrine étant la plus résistante et l'aldrine la plus sensible. 


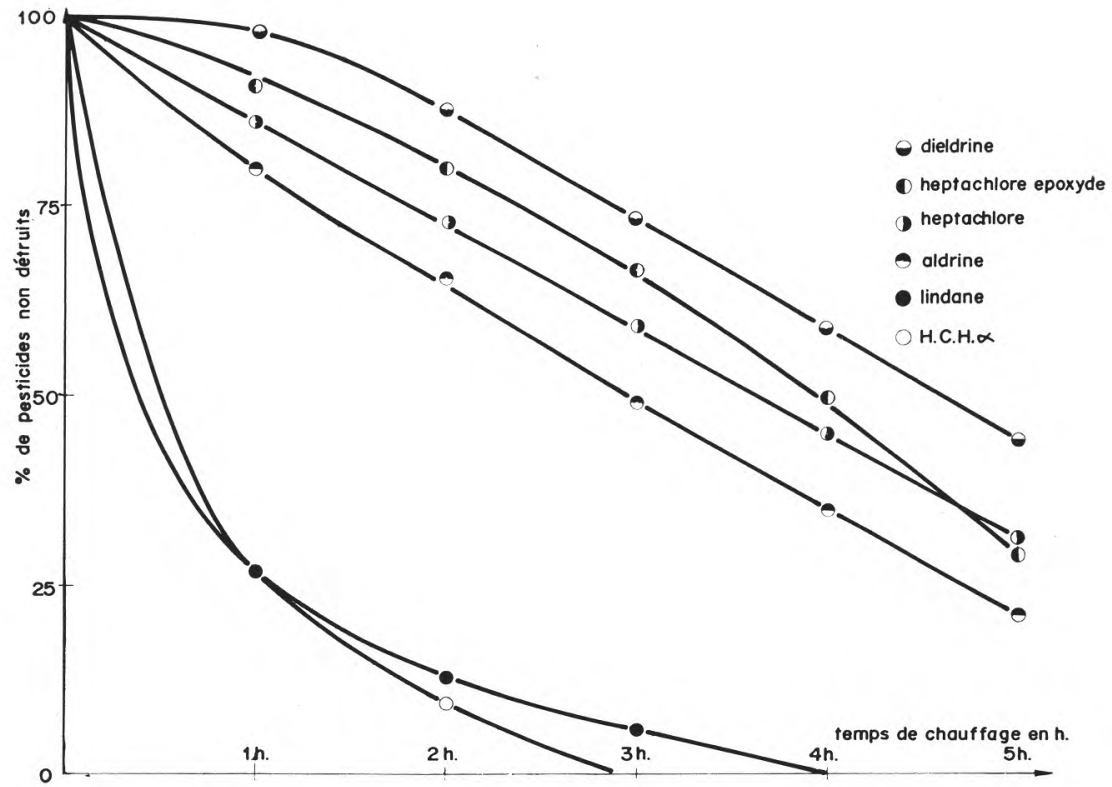

fig. 2

Effets du chauffage à $300^{\circ} \mathrm{C}$

$T A B L E A U 1$

Pourcentage de destruction thermique des hexachlorocyclohexanes

\begin{tabular}{|c|c|c|c|c|c|c|}
\hline \multirow{2}{*}{ Pesticides } & \multirow{2}{*}{$\begin{array}{l}\text { Températures } \\
\text { en }{ }^{\circ} \mathrm{C}\end{array}$} & \multicolumn{5}{|c|}{$\begin{array}{c}\text { Durée du traitement thermique } \\
\text { en heures }\end{array}$} \\
\hline & & 1 & 2 & 3 & 4 & 5 \\
\hline H.C.H. $\alpha$ & $\begin{array}{l}150 \\
200 \\
250 \\
300 \\
350\end{array}$ & $\begin{array}{r}4 \\
6 \\
25 \\
73 \\
100\end{array}$ & $\begin{array}{r}8 \\
11 \\
45 \\
90 \\
100\end{array}$ & $\begin{array}{r}12 \\
16 \\
61 \\
100 \\
100\end{array}$ & $\begin{array}{r}15 \\
23 \\
74 \\
100 \\
100\end{array}$ & $\begin{array}{r}19 \\
26 \\
88 \\
100 \\
100\end{array}$ \\
\hline Lindane & $\begin{array}{l}150 \\
200 \\
250 \\
300 \\
350\end{array}$ & $\begin{array}{r}3 \\
6 \\
17 \\
73 \\
100\end{array}$ & $\begin{array}{r}7 \\
11 \\
29 \\
87 \\
100\end{array}$ & $\begin{array}{r}12 \\
16 \\
40 \\
94 \\
100\end{array}$ & $\begin{array}{r}16 \\
23 \\
52 \\
100 \\
100\end{array}$ & $\begin{array}{r}20 \\
26 \\
63 \\
100 \\
100\end{array}$ \\
\hline
\end{tabular}


Les tableaux 1 et 2 résument l'ensemble des résultats obtenus aux cinq températures appliquées, en pourcent de destruction.

Nous avons fait quelques essais qui nous ont permis de mettre en évidence la formation d'acide chlorhydrique. Une partie du chlore est donc libérée, mais il existe aussi des états intermédiaires de la dégradation que nous n'avons pas étudiés.

L'examen des différentes courbes et du tableau 3 permet d'affirmer qu'il existe en fonction de la température et pour chaque pesticide au moins trois types de vitesse de dégradation. Aux basses températures, la vitesse de dégradation est nulle, puis quand

\section{TABLEAU 2}

Pourcentage de destruction thermique des dérivés chlorés de l'indène et du naphtalène

\begin{tabular}{|c|c|c|c|c|c|c|}
\hline \multirow{2}{*}{ Pesticides } & \multirow{2}{*}{$\begin{array}{c}\text { Températures } \\
\text { en }{ }^{\circ} \mathbf{C}\end{array}$} & \multicolumn{5}{|c|}{$\begin{array}{c}\text { Durée du traitement thermique } \\
\text { en heures }\end{array}$} \\
\hline & & 1 & 2 & 3 & 4 & 5 \\
\hline Heptachlore & $\begin{array}{l}150 \\
200 \\
250 \\
300 \\
350\end{array}$ & $\begin{array}{r}0 \\
0 \\
5 \\
13 \\
-\end{array}$ & $\begin{array}{r}0 \\
0 \\
9 \\
27 \\
75\end{array}$ & $\begin{array}{r}0 \\
0 \\
13 \\
42 \\
86\end{array}$ & $\begin{array}{l}0 \\
2,5 \\
17 \\
55 \\
-\end{array}$ & $\begin{array}{r}0 \\
5 \\
22 \\
70 \\
100\end{array}$ \\
\hline Heptachlore époxyde & $\begin{array}{l}150 \\
200 \\
250 \\
300 \\
350\end{array}$ & $\begin{array}{l}0 \\
2 \\
3,5 \\
8 \\
-\end{array}$ & $\begin{array}{l}0 \\
3,5 \\
10 \\
20 \\
53\end{array}$ & $\begin{array}{l}0 \\
4,5 \\
18 \\
35 \\
70\end{array}$ & $\begin{array}{r}0 \\
7 \\
27 \\
50 \\
-\end{array}$ & $\begin{array}{l}0 \\
8,5 \\
34 \\
70 \\
90\end{array}$ \\
\hline Aldrine & $\begin{array}{l}150 \\
200 \\
250 \\
300 \\
350\end{array}$ & $\begin{array}{r}0 \\
2 \\
3 \\
25 \\
-\end{array}$ & $\begin{array}{l}0 \\
3,5 \\
5 \\
36 \\
70\end{array}$ & $\begin{array}{c}0 \\
4,5 \\
7,5 \\
50 \\
84\end{array}$ & $\begin{array}{c}0 \\
7,5 \\
10 \\
64 \\
-\end{array}$ & $\begin{array}{l}0 \\
9,5 \\
18 \\
77 \\
97\end{array}$ \\
\hline Dieldrine & $\begin{array}{l}150 \\
200 \\
250 \\
300 \\
350\end{array}$ & $\begin{array}{l}0 \\
0 \\
0 \\
2 \\
-\end{array}$ & $\begin{array}{r}0 \\
0 \\
0 \\
14 \\
37\end{array}$ & $\begin{array}{r}0 \\
0 \\
0 \\
28 \\
52\end{array}$ & $\begin{array}{r}0 \\
0 \\
5 \\
41 \\
-\end{array}$ & $\begin{array}{c}0 \\
2,5 \\
7,5 \\
55 \\
75\end{array}$ \\
\hline
\end{tabular}


TABLEAU 3. - Vitesse de dégradation des pesticides en fonction de la température

\begin{tabular}{|c|c|c|c|c|c|c|}
\hline Pesticide & \multicolumn{2}{|c|}{$150^{\circ} \mathrm{C}$} & $200^{\circ} \mathrm{C}$ & $250^{\circ} \mathrm{C}$ & $300^{\circ} \mathrm{C}$ & $350^{\circ} \mathrm{C}$ \\
\hline H.C.H. $\alpha$ & (B) & $=0,040$ & (B) $\mathrm{k}=0,052$ & (C) $\begin{array}{l}\mathrm{k}=0,246 \\
\mathrm{n}=0,53\end{array}$ & (C) $\begin{array}{l}\mathrm{k}=2,240 \\
\mathrm{n}=1,75\end{array}$ & - \\
\hline Lindane & & $(*)$ & (B) $\mathrm{k}=0,052$ & (C) $\begin{array}{l}\mathrm{k}=0,158 \\
\mathrm{n}=0,48\end{array}$ & (C) $\begin{aligned} \mathrm{k} & =1,445 \\
\mathrm{n} & =1,25\end{aligned}$ & - \\
\hline Aldrine & (A) & - & (B) $\mathrm{k}=0,019$ & (B) $\mathrm{k}=0,025$ & (C) $\begin{array}{l}\mathrm{k}=0,195 \\
\mathrm{n}=0,48\end{array}$ & (C) $\begin{array}{l}\mathrm{k}=0,537 \\
\mathrm{n}=0,96\end{array}$ \\
\hline Dieldrine & & - & (A) $t \leqslant 4 h$ & $\begin{array}{l}\text { (A) } t \leqslant 3 h \\
\text { (D) } t>3 h\end{array}$ & (D) $\begin{aligned} \mathrm{k}^{\prime} & =0,178 \\
\mathrm{n}^{\prime} & =0,19\end{aligned}$ & (C) $\begin{array}{l}\mathrm{k}=0,178 \\
\mathrm{n}=0,40\end{array}$ \\
\hline Heptachlore & & - & $\begin{array}{l}\text { (A) } t \leqslant 3 h \\
\text { (D) } t>3 h\end{array}$ & (B) $\mathrm{k}=0,042$ & (B) $\mathrm{k}=0,137$ & (C) $\begin{array}{l}\mathrm{k}=0,596 \\
\mathrm{n}=0,90\end{array}$ \\
\hline Heptachlore époxyde & & - & (D) & $(*)$ & (D) $\begin{array}{l}\mathrm{k}^{\prime}=0,195 \\
\mathrm{n}^{\prime}=0,24\end{array}$ & (C) $\begin{array}{l}\mathrm{k}=0,275 \\
\mathrm{n}=0,58\end{array}$ \\
\hline
\end{tabular}
(A) $\mathrm{V}=\mathrm{o}$
(B) $\mathrm{V}=\mathrm{k}$
(C) $\mathrm{V}=\mathrm{kcn}$
(D) $\mathrm{V}=\mathrm{k}^{\prime}\left(1-\frac{\mathrm{c}}{\mathrm{c}_{\mathrm{o}}}\right) \mathrm{n}^{\prime}$
(*) non déterminé, courbes inexploitables 
la température augmente, elle est une fonction du produit dégradé, c'est-à-dire de la forme : $\mathrm{v}=\mathrm{k}^{\prime}\left(1-\frac{\mathrm{c}}{\mathrm{c}_{\mathrm{o}}}\right) \mathrm{n}^{\prime}$. Si la température augmente encore, la vitesse devient constante $(\mathrm{v}=\mathrm{k})$, puis suit la loi $\mathrm{v}=\mathrm{kcn}$.

La signification des symboles est la suivante :

$$
\begin{array}{ll}
\mathrm{v} & =\text { vitesse de dégradation instantanée } \\
\mathrm{c} & =\text { concentration instantanée } \\
\mathrm{c}_{0} & =\text { concentration initiale } \\
\mathrm{n} \text { et } \mathrm{n}^{\prime} & =\text { ordres des réactions } \\
\mathrm{k} \text { et } \mathrm{k}^{\prime} & =\text { constantes de vitesse }
\end{array}
$$

\section{2) Rayonnement ultra-violet}

La stabilité des différents pesticides soumis aux rayons U.V. est représentée sur la figure 3. Ce sont les dérivés du cyclohexane qui sont les plus stables et les dérivés du méthanoindène et du diméthanonaphtalène qui le sont le moins. Ceci peut s'expliquer par le fait

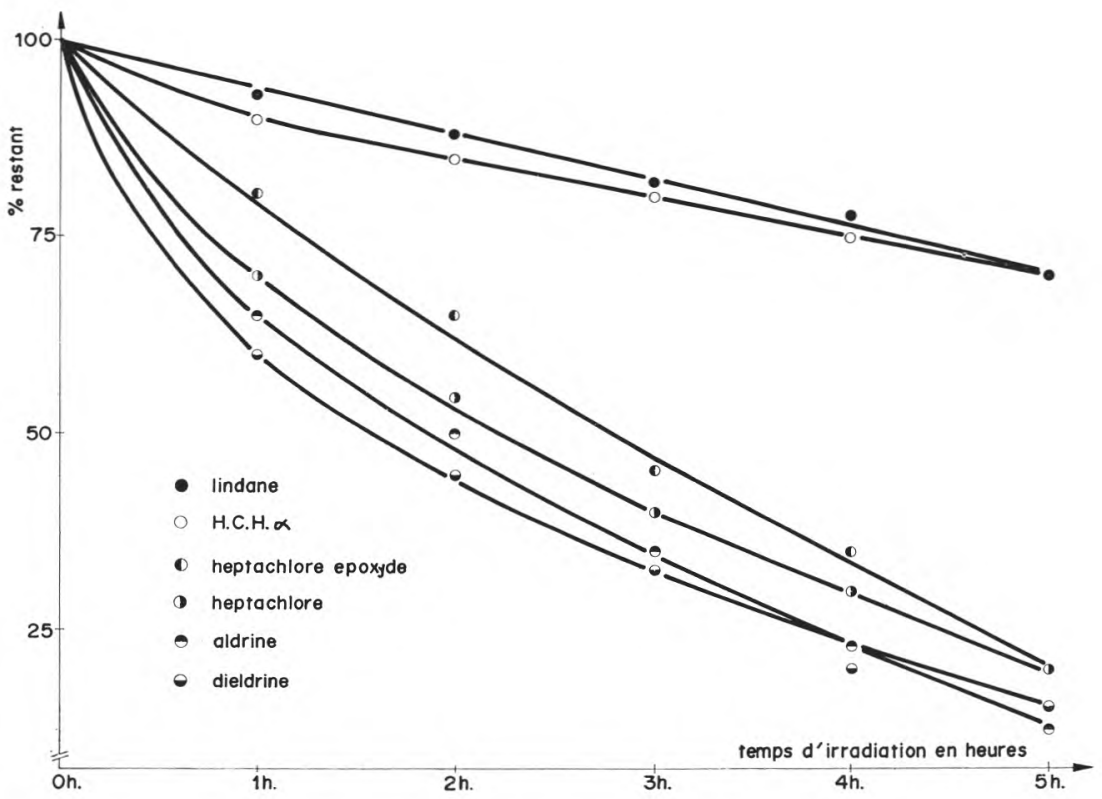

fig. 3

Effet du rayonnement U.V. (254 nm) 
que, pour être dégradé par un rayonnement à une longueur d'onde donnée, un composé doit absorber ce rayonnement. Nous avons enregistré les spectres de ces pesticides dans l'ultra-violet ; ce sont l'H.C.H. $\alpha$ et le lindane qui absorbent le plus faiblement à $254 \mathrm{~nm}$.

\section{3) L'eau oxygénée}

Les pesticides organochlorés ajoutés au lait sont partiellement dégradés par la solution d'eau oxygénée dans les proportions suivantes:
H.C.H. $\alpha$ et $\beta$ : 15 p. 100
H.C.H. $\gamma$ (ou lindane) : 18 p. 100
Heptachlore époxyde : 28 p. 100
Dieldrine
: 45 p. 100

L'heptachlore époxyde et la dieldrine sont les composés de notre série les plus sensibles à l'action de l'eau oxygénée.

\section{4) Le stockage}

Le verre pyrex arrête les radiations de longueur d'onde inférieure à $360 \mathrm{~nm}$; les échantillons placés à la lumière ont donc peu subi l'action des rayons U.V. solaires (396 à $292 \mathrm{~nm}$ ). Cependant, un effet photochimique est évident pour l'aldrine. Le tableau 4 montre que les pesticides organochlorés présentent des stabilités très variables. Aucune dégradation de l'H.C.H. , du lindane et de la dieldrine n'a pu être mise en évidence. L'aldrine est le composé le moins stable et le seul à être sensible aux conditions de stockage. Nous n'avons pas constaté l'apparition d'heptachlore époxyde lors de la disparition de l'heptachlore, par contre, des traces de dieldrine ont été décelées dans les échantillons contenant de l'aldrine et conservés à la lumière.

\section{DISCUSSION}

Nos essais confirment la résistance des pesticides organochlorés aux traitements thermiques et nos résultats concordent avec ceux de Kroger [5] qui a travaillé avec une gamme de substances différentes; cependant, cette résistance n'est pas uniforme; les dérivés $\mathrm{du}$ cyclohexane sont les plus sensibles au chauffage. Il en va différemment avec le traitement aux rayons U.V. ; dans ce cas ce sont les dérivés du méthanoindène et du diméthanonaphtalène qui sont les plus rapidement détruits.

En ce qui concerne les produits de dégradation obtenus par irradiation à $254 \mathrm{~nm}$, nous n'avons pas pu déceler l'apparition de dieldrine (à partir de l'aldrine) ou de différents photoisomères, comme l'avaient fait Keith et Alford [7]. Par contre, dans les 
TABLEAU 4. - Stabilité des pesticides organochlorés dans le temps (p. 100 résiduels)

\begin{tabular}{|c|c|c|c|c|c|}
\hline \multicolumn{2}{|c|}{$\begin{array}{l}\text { Conditions de stockage } \\
\text { durée }\end{array}$} & $\begin{array}{l}\text { Dieldrine } \\
\text { H.C.H. } \alpha \\
\text { Lindane }\end{array}$ & Heptachlore & $\begin{array}{l}\text { Heptachlore } \\
\text { époxyde }\end{array}$ & Aldrine \\
\hline $\begin{array}{l}12 \text { mois } \\
12 \text { mois } \\
12 \text { mois }\end{array}$ & $\begin{array}{l}22^{\circ} \mathrm{C} \text { lumière } \\
22^{\circ} \mathrm{C} \text { obscurité } \\
6^{\circ} \mathrm{C} \text { obscurité }\end{array}$ & $\begin{array}{l}100 \\
100 \\
100\end{array}$ & $\begin{array}{l}87 \\
87 \\
87\end{array}$ & $\begin{array}{l}100 \\
100 \\
100\end{array}$ & $\begin{array}{l}18 \\
37 \\
50\end{array}$ \\
\hline $\begin{array}{l}20 \text { mois } \\
20 \text { mois } \\
20 \text { mois }\end{array}$ & $\begin{array}{l}22^{\circ} \mathrm{C} \text { lumière } \\
22^{\circ} \mathrm{C} \text { obscurité } \\
6^{\circ} \mathrm{C} \text { obscurité }\end{array}$ & $\begin{array}{l}100 \\
100 \\
100\end{array}$ & $\begin{array}{l}78 \\
78 \\
78\end{array}$ & $\begin{array}{l}90 \\
90 \\
90\end{array}$ & $\begin{array}{r}8 \\
27 \\
42\end{array}$ \\
\hline $\begin{array}{l}28 \text { mois } \\
28 \text { mois } \\
28 \text { mois }\end{array}$ & $\begin{array}{l}22^{\circ} \mathrm{C} \text { lumière } \\
22^{\circ} \mathrm{C} \text { obscurité } \\
6^{\circ} \mathrm{C} \text { obscurité }\end{array}$ & $\begin{array}{l}100 \\
100 \\
100\end{array}$ & $\begin{array}{l}70 \\
70 \\
70\end{array}$ & $\begin{array}{l}80 \\
80 \\
80\end{array}$ & $\begin{array}{r}0 \\
15 \\
35\end{array}$ \\
\hline
\end{tabular}


expériences de stockage de la graisse de beurre, nous avons mis en évidence des traces de dieldrine présentes dans des échantillons contenant de l'aldrine et exposés à la lumière solaire.

L'eau oxygénée a un effet destructeur important ; c'est de plus un bactéricide efficace. Rappelons que son emploi est interdit en France, mais autorisé dans certains pays, en particulier aux EtatsUnis, afin d'améliorer la qualité du lait pour la fabrication de fromages à pâte cuite ; son action étant suivie d'un traitement à la catalase $[10,11]$. En ce qui concerne les pourcentages de destruction, nos résultats s'accordent avec ceux de Heckmati et Bradley [8].

\section{R és u mé}

Les traitements thermiques de $150^{\circ} \mathrm{C}$ à $350^{\circ} \mathrm{C}$, appliqués aux pesticides organochlorés pendant des temps variant de $1 \mathrm{~h}$ à $5 \mathrm{~h}$, dégradent surtout les dérivés du cyclohexane. Une étude cinétique permet de montrer l'existence de trois types de vitesse de dégradation en fonction de la température. Les rayons U.V. détruisent plus rapidement les dérivés du méthanoindène et du diméthanonaphtalène que les dérivés du cyclohexane.

L'eau oxygénée ajoutée au lait, à raison de 0,03 p. 100 en poids, a une action destructive sur tous les insecticides éprouvés; le pourcentage de destruction va de 15 pour les cyclohexanes à 45 pour la dieldrine. Le stockage de la graisse de beurre jusqu'à 28 mois, dans différentes conditions, ne dégrade pas le cyclohexane ni la dieldrine; par contre, l'heptachlore, l'heptachlore époxyde et l'aldrine subissent une dégradation assez importante.

\section{S u m m a r y}

Heating of organochlorine pesticides from 150 to $350^{\circ} \mathrm{C}$ degrades mainly the cyclohexane derivatives. A kinetical study has shown the existence of three kinds of degradation rate by function of temperature. The ultra violet energy destroys the methanoindene and dimethanonaphtalene derivatives more quickly that the cyclohexane derivatives.

The destroying action of hydrogen peroxyde is important (from 15 p. 100 for cyclohexane to 45 p. 100 for dieldrine). During storage for long time the cyclohexanes are less degradeted than others chlorinated pesticides. 


\section{Bibliographie}

[1] Langlois (B. E.), Liska (B. J.) and Hill (D. L.) (1964). - The effect of processing and storage of dairy products on chlorinated insecticide residues. J. Milk Food Technol., 27, 264-267.

[2] Langlois (B. E.), Liska (B. J.) and Hill (D. L.) (1965). - The effect of processing and storage of dairy products on chlorinated insecticide residues. J. Milk Food Technol., 28, 9-11.

[3] Li (C. F.), Bradley (R. L.) and Schultz (L. H.) (1970). - Fate of organochlorine pesticides during processing of milk into dairy products. J. Assoc. Offic. Anal. Chem., 53, 127-139.

[4] Ledford (R. A.), CHEN (J. H.) and SHIPE (W. F.) (1968). - Effect of direct steam heating and vacuum treatment on levels of pesticide residues in milk. J. Dairy Sci., 51, 219-220.

[5] Kroger (M.) (1968). - Effect of various physical treatments on certains organochlorine hydrocarbon insecticides found in milk fat. J. Dairy Sci., 51, 194-198.

[6] Li (L. F.) and Bradley (R. L.) (1969). - Degradation of chlorinated hydrocarbon pesticides in milk and butter oil by ultra-violet energy. J. Dairy Sci., 52, 27-30.

[7] Keith (L. H.) and Alford (A. L.) (1970). - Review of the application of Nuclear Magnetic Resonnance Spectroscopy in pesticide analysis. J. Assoc. Offic. Anal. Chem., 1018-1035.

[8] Hekmati (M.) and Bradley (R. L.) (1971). - Degradation of organochlorine pesticides with hydrogen peroxyde. Milchwissenschaft, 26, 224-226.

[9] Langlois (B. E.), Stemp (A. R.) and Liska (B. J.) (1964). - Rapid Clean up of dairy products for analysis of chlorinated insecticides residues by electron capture gas chromatography. J. Agr. Food. Chem., 12, 243-250.

[10] Code of Federal Regulation (1966). 21 CFR 19500 E3 : 165.

[11] FAO (1957). Report on the Meeting of Experts on the Use of Hydrogen Peroxyde and other Preservatives in Milk, FAO/57/11/8655. 